\title{
Pattern Synthesis of Planar Nonuniform Circular Antenna Arrays Using a Chaotic Adaptive Invasive Weed Optimization Algorithm
}

\author{
Huaning Wu, Chao Liu, and Xu Xie \\ Department of Electronic Engineering, Naval University of Engineering, Wuhan 430033, China \\ Correspondence should be addressed to Huaning Wu; wuhuaning007@163.com
}

Received 17 February 2014; Revised 7 May 2014; Accepted 21 May 2014; Published 19 June 2014

Academic Editor: Fang Zong

Copyright (c) 2014 Huaning Wu et al. This is an open access article distributed under the Creative Commons Attribution License, which permits unrestricted use, distribution, and reproduction in any medium, provided the original work is properly cited.

A novel invasive weed optimization (IWO) variant called chaotic adaptive invasive weed optimization (CAIWO) is proposed and applied for the optimization of nonuniform circular antenna arrays. A chaotic search method has been combined into the modified IWO with adaptive dispersion, where the seeds produced by a weed are dispersed in the search space with standard deviation specified by the fitness value of the weed. To evaluate the performance of CAIWO, several representative benchmark functions are minimized using various optimization algorithms. Numerical results demonstrate that the proposed approach improves the performance of the algorithm significantly, in terms of both the convergence speed and exploration ability. Moreover, the scheme of CAIWO is employed to find out an optimal set of weights and antenna element separation to obtain a radiation pattern with maximum side-lobe level (SLL) reduction with different numbers of antenna element under two cases with different purposes. The design results obtained by CAIWO have comfortably outperformed the published results obtained by other state-of-the-art metaheuristics in a statistically meaningful way.

\section{Introduction}

In several applications, such as mobile communication and spatial detection, techniques require antennas that have high directive radiation pattern, which cannot be achieved by a single element antenna. Antenna arrays are formed to circumvent such problems by combining many individual antenna elements in particular electrical and geometrical configurations. The primary design objective of antenna array geometry is to determine the locations of array elements that jointly produce a radiation pattern to resemble the desired pattern as nearly as possible. Poor design may result in a polluted electromagnetic environment. This will also result in wastage of power, which is a vital aspect in wireless devices that run on batteries. The classical derivative-based optimizations of designing antenna arrays are not effective as they are prone to getting local optima and strongly sensitive to initialization. Due to these inherent shortcomings of the classical technique, many modern metaheuristics approaches were tried to achieve optimized side-lobe level (SLL) and null control from the designed arrays [1-6].

Circular arrays have become popular in recent years over array over other array geometries because they have the capability to perform the scan in all directions without a considerable change in the beam pattern and provide $360^{\circ}$ azimuth coverage [7]. Moreover, circular arrays are less sensitive to mutual coupling as compared to linear and rectangular arrays since these do not have edge elements [8]. Circular antenna arrays are used in various applications in radar, sonar, mobile, and commercial satellite communication systems. The first metaheuristic approach towards the design circular arrays is real-coded genetic algorithm (GA), which can be traced in the work of Panduro et al. [9]. Later, particle swarm optimization was applied by Shihab et al. in [10] for the design of circular array. Panduro et al. [11] compared three powerful population-based optimization 
algorithms PSO, GA, and differential evolution (DE) on the design problem of scanned circular arrays. Gürel and Ergül applied GA in [12] to design a circular array where each element was log-periodic antenna. In literature [13], Roy et al. applied a modified IWO algorithm to design a nonuniform circular antenna arrays. Some other applications of metaheuristics for the design of circular antenna array are found in $[7,14-17]$ that involves the use of biogeography based optimization (BBO), simulated algorithm (SA), and so on.

Amongst all evolutionary algorithms (EAs) described in various articles, invasive weed optimization has emerged as one of the most powerful tools for solving the real world optimization problems [18]. Invasive weed optimization has been successfully applied to solve problems in electromagnetic as found in literature [19-26]. As IWO is a stochastic search process hence it is not free from false or untimely convergence, in particular over multimodal fitness landscapes. To eradicate this problem, IWO needs to be modified. In this paper, we present here a new powerful variant of IWO denoted by CAIWO, for designing nonuniform circular arrays with optimized performance with respect to SLL, dynamic range ratios, beam width, directivity, and null control in a scanning range $\left[0^{\circ}, 360^{\circ}\right]$. Comparisons with the other well-known optimizers like GA [9], PSO [10], DE [15], BBO [7], original IWO and MIWO [13] have been made to verify the search ability of the proposed algorithm.

The rest of the paper is organized an follows: Section 2 gives a brief overview of the classical IWO algorithm and a comprehensive overview of the proposed CAIWO algorithm. A formulation of the array pattern synthesis as an optimization task has been discussed in Section 3. Section 4 presents the simulation results and in Section 5 conclusions are presented.

\section{The Iwo Algorithm and Its Modification}

2.1. Description of Traditional IWO. The original IWO algorithm was initially proposed by Mehrabian and Lucas in [18] for solving continuous optimization problems. IWO mimics the process of colonizing and distributing behavior of weeds. The IWO algorithm may be summarized as four steps, more details can be found in $[18,20]$.

(I) Initialization. Solutions are initialized and dispersed in the given $n$-dimensional search space uniformly and randomly.

(II) Reproduction. Each member of the population is allowed to produce seeds depending on its own, as well as the colony's lowest and highest fitness, so that the number of seeds produced by a weed increases linearly from lowest possible seed for a weed with worst fitness to the maximum number of seeds for a plant with best fitness.

(III) Spatial Distribution. The generated seeds are randomly scattered over the $d$-dimensional search space by perturbing them with normally distributed random numbers with zero

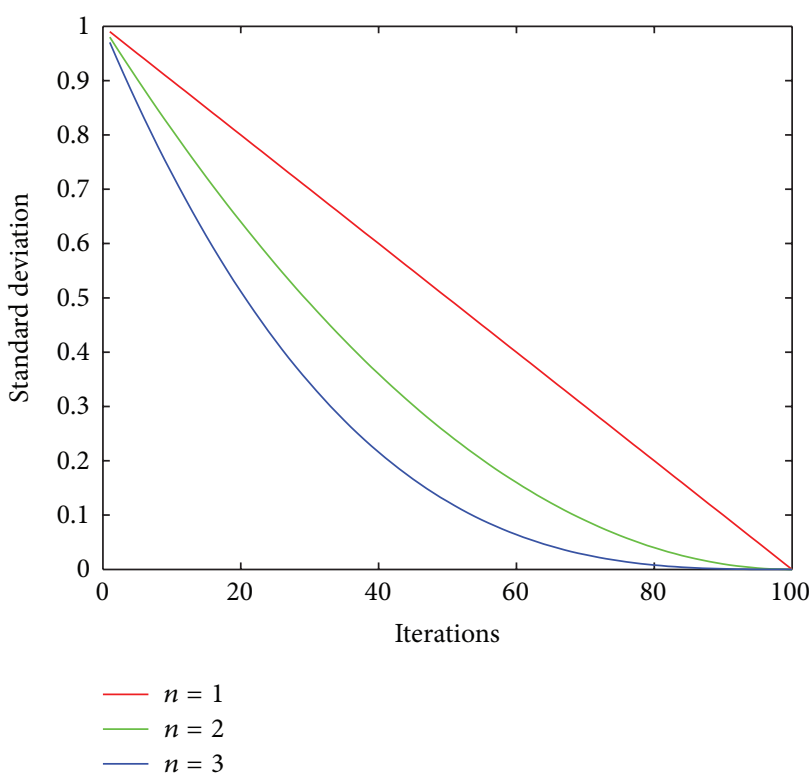

FIGURE 1: Standard deviation over the course of the run.

mean and a variable variance. The standard deviation for a particular iteration can be given as follows:

$$
\delta_{\text {cur }}=\frac{\left(\text { iter }_{\text {max }}-\text { iter }\right)^{n}}{\text { iter }_{\text {max }}^{n}}\left(\delta_{\text {initial }}-\delta_{\text {final }}\right)+\delta_{\text {final }},
$$

where $\delta_{\text {initial }}$ and $\delta_{\text {final }}$ represent the initial and final standard deviations (SD) and iter ${ }_{\max }$ and iter are the maximum number of iteration and the current iteration number respectively. $n$ is called the nonlinear modulation index. This is a relatively critical parameter which can influence the convergence performance of the IWO. Through a set of simulations, it has been shown that the best choice for $n$ is 3. Figure 1 shows the standard deviation over the course of a run with 100 iterations and different modulation indexes. Having selected this suitable value for nonlinear modulation index, the algorithm starts with a relatively high SD that the optimizer can scan of the solution space completely. As the iteration number increases and SD value is decreased, the search would be restricted to the neighborhoods around the local minima or maxima to find the global optimal solution.

Then, the position of the new seed can be given as follows:

$$
x_{\text {son }}=x_{\text {parent }}+s d=x_{\text {parent }}+\operatorname{rand} n(0,1) * \delta_{\text {cur }} .
$$

(IV) Competitive Exclusion. Some kinds of competition between plants are needed for limiting maximum number of plants in a colony. Initially, the plants in a colony will reproduce fast and all the produced plants will be included in the existing colony, until the number of plants in the colony reaches a maximum value $p_{\max }$. The steps (I) to (IV) are repeated until the maximum number of iterations has reached; that is, the colony size is fixed from thereon to $p_{\max }$.

2.2. The Features and Shortcomings of IWO. One important advantage of the IWO is that it allows all of possible candidates to participate in the reproduction process. From step 
TABLE 1: Test functions.

\begin{tabular}{lcc}
\hline Function name & Search range & Dimension \\
\hline Sphere & {$[-100,100]$} & 30 \\
Rastrigin & {$[-5.12,5.12]$} & 30 \\
Ackley & {$[-32,32]$} & 30 \\
Griewank & {$[-600,600]$} & 30 \\
\hline
\end{tabular}

(II) in Section 2.1, we can know that the fitter plants produce more seeds than less fit plants, which tends to improve the convergence of the algorithm. In contrast, most metaheuristic algorithms would not allow the less fit plants to produce offspring such as the GA. Another important property of IWO is straightforward and it includes less deal computational burden unlike other methods such as PSO. PSO needs to update both the position and velocity of individuals in each iteration which cost some extra computations to search the best position in the neighborhood of each particle as well as the whole population. Finally, the weeds in IWO can produce seeds without mating so that each agent may have different number of variables during the optimization process. Thus, the number of variables can be chosen as one of the optimization parameters in this algorithm [20].

But there are also some shortcomings of IWO, the most prominent one is concerning the way the seeds produced by a plant are dispersed in the search spaces. As mentioned in Section 2.1, we can know that the function of SD defines the exploration ability and exploitation ability of algorithm and acts as both diversification and intensification components of IWO; it has a great effect on final solutions. In early iterations, the bigger SD will help the algorithm to explore the solution space as much as it can. A good diversification will make the final solution near to global optimum. The algorithm will use this component to identify most the potential spaces where the global optimum may lie in. A good intensification will help the algorithm to exploit the potential areas to find the global optimum. It will increase the convergence speed of the algorithm and search the better final solution. Hence, it is very important to keep an efficient balance between diversification and intensification of the algorithm. But, IWO algorithm uses a fix SD to produce seeds related to each weed, and suffers from the lack of fine balance between exploration and exploitation.

2.3. Chaotic Adaptive Invasive Weed Optimization. In order to overcome the drawbacks of IWO, a chaotic search and adaptive dispersion mechanism are integrated into the IWO algorithm. This variant IWO is named CAIWO.

2.3.1. Chaotic Search. Chaotic search methods have a greater ability to escape from the local minima. Therefore, the CAIWO algorithm has a less chance of premature convergence compared to original IWO. There are many chaotic maps such as logistic map, sinusoidal map, and tent map. We chose the sinusoidal map to improve the performance of IWO based on the discussion in the literature [27].

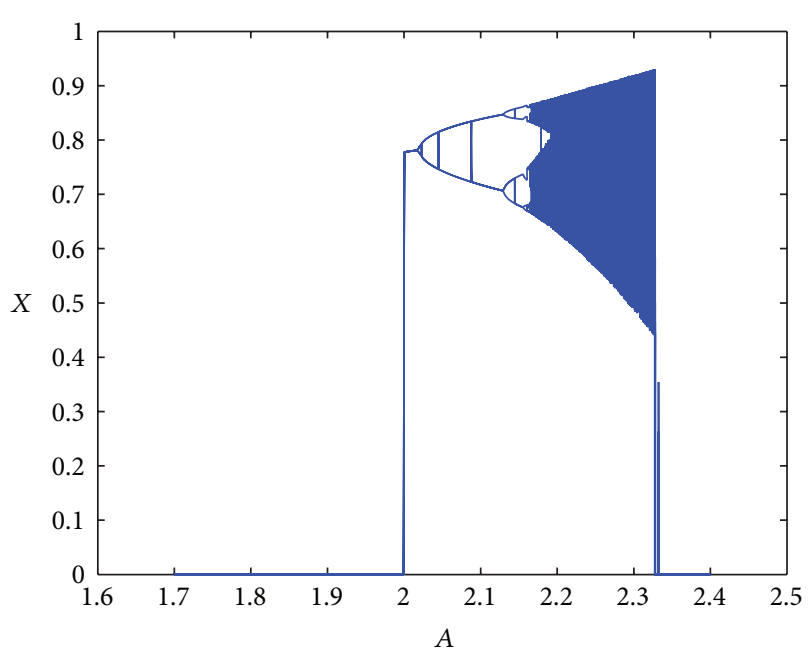

Figure 2: The bifurcation diagram for sinusoidal map.

The sinusoidal map or the sinusoid sequence is defined by

$$
X_{k+1}=A X_{k}^{2} \sin \left(\pi X_{k}\right)
$$

which ensures chaotic behavior in the span of $(0,1)$. Figure 2 shows the variations of the chaotic variable versus changes in the control parameter. As it can be seen, the performance of the system becomes chaotic when $A=2.3$.

2.3.2. Adaptive Dispersion. The adaptive dispersion mechanism is that the SD of the current generation distribute linearly among the weeds as weed with the highest fitness achieves the lowest SD and the lowest fitness achieves highest $\mathrm{SD}$, which can be represented by (4). $j$ is the index of weeds in the colony which sorted according to their fitness, $\sigma_{\text {current }}$ can be calculated by (1), $p_{\text {sum }}$ is the sum number of weeds in the current generation, and $\sigma_{j}$ is the SD of $j$ th weeds to produce seeds. Hence, the plant with lower fitness will have the chance to produce good seeds in current generation. In addition, this concept will increase the diversification of algorithm so the algorithm will explore the search space effectively. Consider

$$
\sigma_{j}=\left(\sigma_{\text {current }}\right) \times\left(\frac{j}{p_{\text {sum }}}\right) .
$$

2.3.3. CAIWO. The goal of the optimization algorithm is to minimize $F\left(X_{1}, X_{2}, \ldots, X_{D}\right)$ subject to $X_{\min }^{i}<X_{i}<$ $X_{\max }^{i}$, where $i=1,2, \ldots, D$ and $D$ is the dimension of the problem. A plant in the CAIWO represents a solution $X=\left(X_{1}, X_{2}, \ldots, X_{D}\right)$ in the real problem. The steps of the proposed CAIWO algorithm proceed as follows.

(1) Initially, pioneering solutions are initialized in the search space range of $\left(X_{\min }, X_{\max }\right)$. Chaotically distribute the pioneering solutions using the sinusoidal map described in Section 2.3.1. It is worth noting that the variables should be normalized to the range of $(0,1)$ before applying the chaotic map. The normalization procedure is described as follows. 
TABLE 2: Simulation results on benchmark functions.

\begin{tabular}{|c|c|c|c|c|c|}
\hline Benchmark function & Algorithm & Fitness of best run & Fitness of worst run & Average value & Standard deviation \\
\hline \multirow{3}{*}{ Sphere function } & IWO & $2.6 E-05$ & $7.11 E-05$ & $3.97 E-05$ & $5 E-6$ \\
\hline & MIWO & $7.93 E-06$ & $2.44 E-05$ & $1.40 E-05$ & $2.00 E-06$ \\
\hline & CAIWO & $1.65 E-10$ & $1.93 E-09$ & $7.14 E-10$ & $1.62 E-10$ \\
\hline \multirow{3}{*}{ Rastrigin function } & IWO & 0.044136 & 0.994962 & 0.797145 & 0.154781 \\
\hline & MIWO & 0.095132 & 1.241246 & 0.872624 & 0.139673 \\
\hline & CAIWO & $1.78 E-06$ & $1.34 E-05$ & $8.12 E-5$ & $1.87 E-6$ \\
\hline \multirow{3}{*}{ Ackley function } & IWO & 0.083654 & 0.099234 & 0.087016 & 0.001684 \\
\hline & MIWO & 0.085449 & 0.256521 & 0.854496 & 0.256521 \\
\hline & CAIWO & $1.88 E-05$ & $9.51 E-05$ & $6.5 E-05$ & $3.12 E-6$ \\
\hline \multirow{3}{*}{ Griewank function } & IWO & 0.049237 & 0.150065 & $1.02 E-01$ & $1.01 E-02$ \\
\hline & MIWO & 0.024603 & 0.113173 & $8.93 E-02$ & $2.22 E-02$ \\
\hline & CAIWO & $1.18 E-10$ & $9.74 E-10$ & $6.57 E-10$ & $4.74 E-12$ \\
\hline
\end{tabular}

TABLe 3: Problem description.

\begin{tabular}{lcc}
\hline Problem number & Numbers of array elements & FNBW \\
\hline 1 & 8 & 70.27 \\
2 & 10 & 55.85 \\
3 & 12 & 46.26 \\
\hline
\end{tabular}

(I) Transform variable $X$ to $\bar{X}$ confine in the data range $(0,1)$ :

$$
\bar{X}=\frac{X-X_{\min }}{X_{\max }-X_{\min }} .
$$

(II) Apply the sinusoidal sequence to transform $\bar{X}$ to a new value $\bar{X}^{\prime}$.

(III) Transform $\bar{X}^{\prime}$ into the range $\left(X_{\min }, X_{\max }\right)$ by

$$
X=X_{\min }+\bar{X}\left(X_{\max }-X_{\min }\right) .
$$

(2) Evaluate each weed, sort, and rank them according to their fitness in the colony.

(3) Sum the number of current generation and calculate the SD of each weed with respect to theirs ranking in the colony using (4). And then new seeds are dispersed randomly on the field with the adaptive SD.

(4) Distribute the newly generated seeds using sinusoidal map in the neighborhood of the parent weed. If the chaotically distributed seed has a better fitness than the previous seed, keep the better one. Otherwise, the chaotic search is continued. The algorithm is guaranteed to converge much faster by taking advantage of the local search superiorities of chaotic search.

(5) Rank the seeds again and exclude those with lower fitness to reach the maximum number of seeds $p_{\max }$.

(6) Continue from step 3 until maximum number of iterations is reached or a criterion is satisfied.

2.4. Simulation of CAIWO to Benchmark Functions. To verify its effectiveness, CAIWO has been applied to classical benchmark functions. All simulations are conducted in a Windows
7 Professional OS using 12-core processors with Intel Xeon $(R), 3.33 \mathrm{GHz}, 72 \mathrm{~GB}$ RAM, and the codes were implemented in MATLAB 7.10. In this section, four benchmark functions including unimodal and multimodal functions in from the test suite of the Congress on Evolutionary Computation (CEC) 2005 Special Session and Competition on Real Parameter Optimization [28] are employed. Test functions, search range $\left(X_{\min }, X_{\max }\right)$, and dimension for functions are listed in Table 1. All the benchmarks functions have been tested for 30 dimensions. Each experiment was carried out with 1000 iterations for the maximum population size of 50. It is a wellknown fact that a stochastic optimization algorithm does not get same results over repeated runs on the same problem. So, we report the mean and the standard deviation of the bestof-run values for 30 independent runs of each of the three algorithms.

$$
\begin{aligned}
& \text { Sphere function: } f_{1}=\sum_{i=1}^{n} x_{i}^{2} \text {. } \\
& \text { Rastrigin function: } f_{2}=\sum_{i=1}^{n}\left(x_{i}^{2}-10 \cos \left(2 \pi x_{i}\right)+10\right) \text {. } \\
& \text { Ackley function: } f_{3}=-20 * \exp (-0.2 * \\
& \left.\sqrt{(1 / n) \sum_{i=1}^{n} x_{i}^{2}}\right)-\exp \left((1 / n) \sum_{i=1}^{n} \cos \left(2 \pi x_{i}\right)\right)+20+ \\
& \exp (1) \text {. } \\
& \text { Griewank function: } f_{4}=\sum_{i=1}^{n}\left(x_{i}^{2} / 4000\right)- \\
& \prod_{i=1}^{n} \cos \left(x_{i} / \sqrt{i}\right)+1 .
\end{aligned}
$$

The performance of CAIWO is compared with MIWO and classical IWO. The simulation results such as fitness of best run, fitness of worst run, and the standard deviation of fitness are shown in Table 2. The convergence of mean fitness values of benchmark functions is shown in Figure 3. From Table 2 and Figure 3, we can see that the CAIWO algorithm obviously performs better than the other two algorithms. It converges successfully and quickly in all functions.

\section{Formulation of the Design Problem}

The $N$-element circular array is shown in Figure 4 . The elements are nonuniformly spaced on a circle of radius $r$ in the $x-y$ plane. The elements are assumed to be isotropic sources so that the radiation pattern of this array can be 

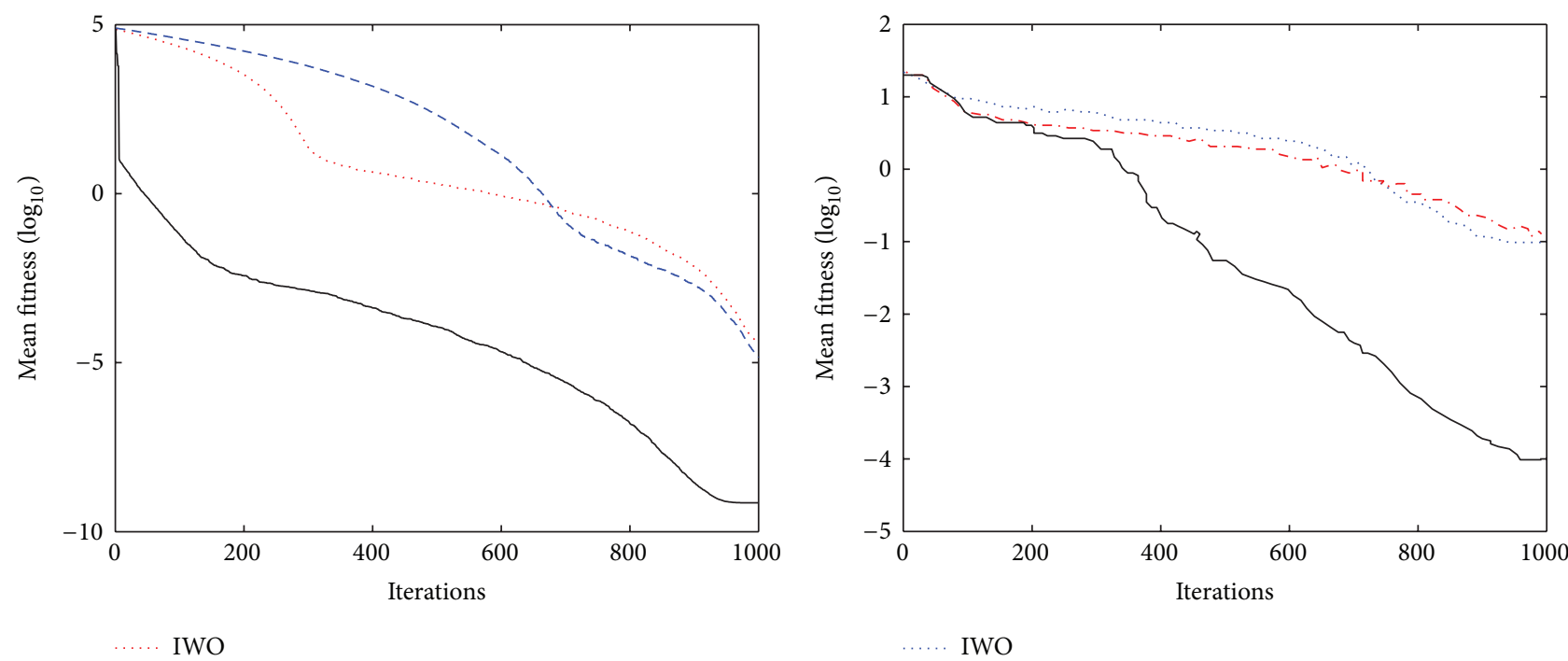

- - - MIWO

— CAIWO

IWO

-..- MIWO

- CAIWO

(a)

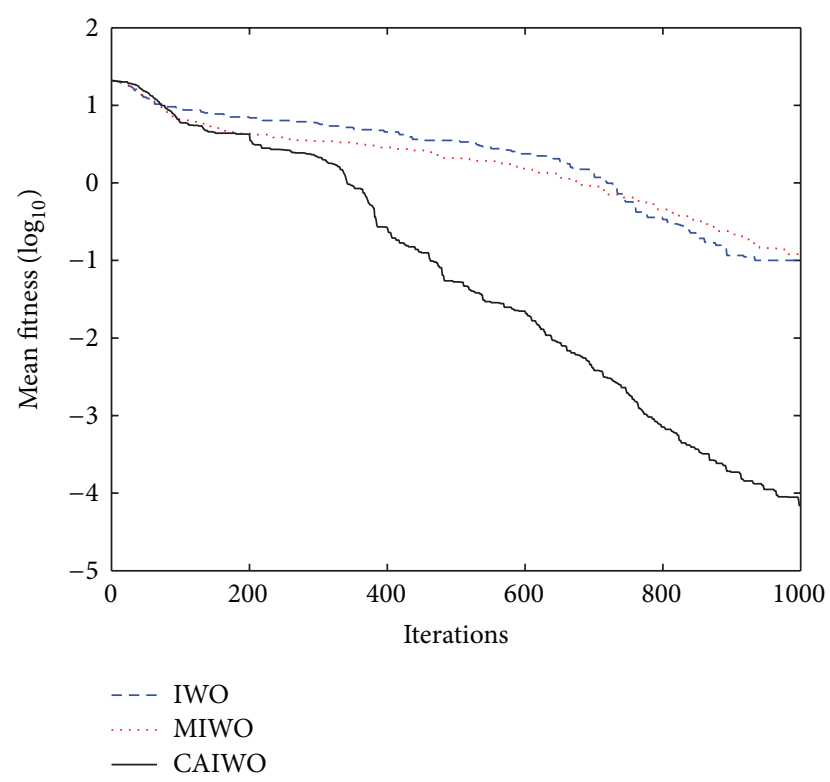

(c)

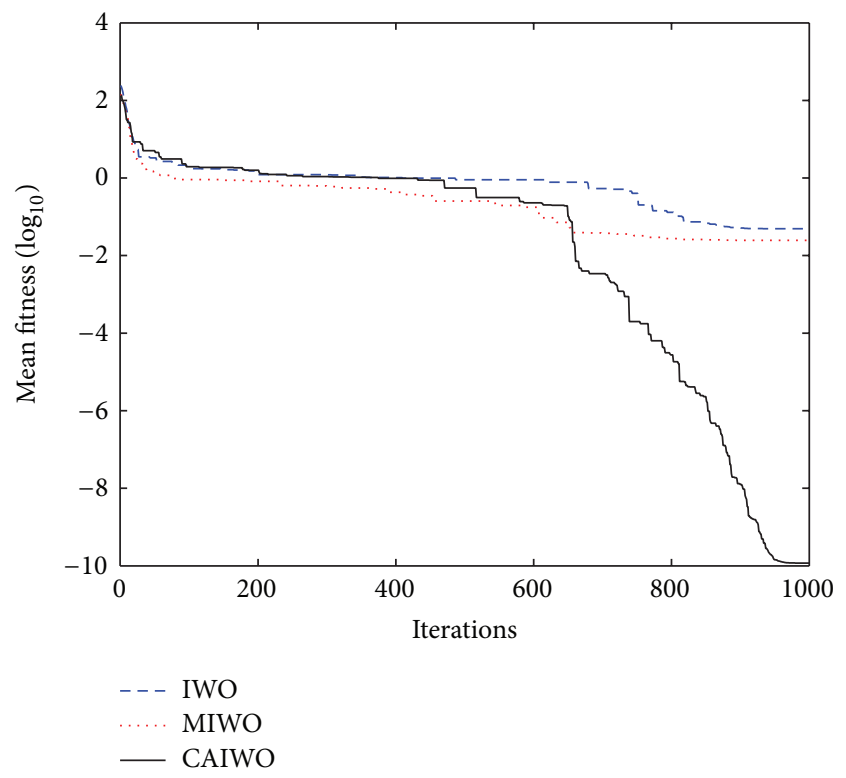

(d)

FIGURE 3: Convergence characteristic of IWO, MIWO, and CAIWO over four benchmarks. (a) Sphere. (b) Rastrigin. (c) Ackley. (d) Griewank.

described by its array factor. The formulation of the array factor requires the following: $a_{n}$ is the normalized amplitude excitation; $\psi_{n}$ is the phase excitation of the $n$th element; $\phi_{n}$ is the angular position of the $n$th element; the circular arc separation between any two adjacent elements $d_{n}$.

The array factor in the $x-y$ plane can be written by [13]

$$
A F(\phi)=\sum_{n=1}^{N} a_{n} \cdot e^{j\left(k r \cos \left(\phi-\phi_{n}\right)+\psi_{n}\right)} .
$$

$k r$ and $\phi_{n}$ can be given by

$$
\begin{gathered}
k r=\frac{2 \pi r}{\lambda}=\sum_{i=1}^{N} d_{i}, \\
\phi_{n}=\frac{2 \pi}{k r} \sum_{i=1}^{n} d_{i} .
\end{gathered}
$$

When the peak of the array is in $\phi_{0}$ direction, the excitation phase of the $n$th element can be written as [13]

$$
\psi_{n}=-k r \cdot \cos \left(\phi_{0}-\phi_{n}\right) \text {. }
$$




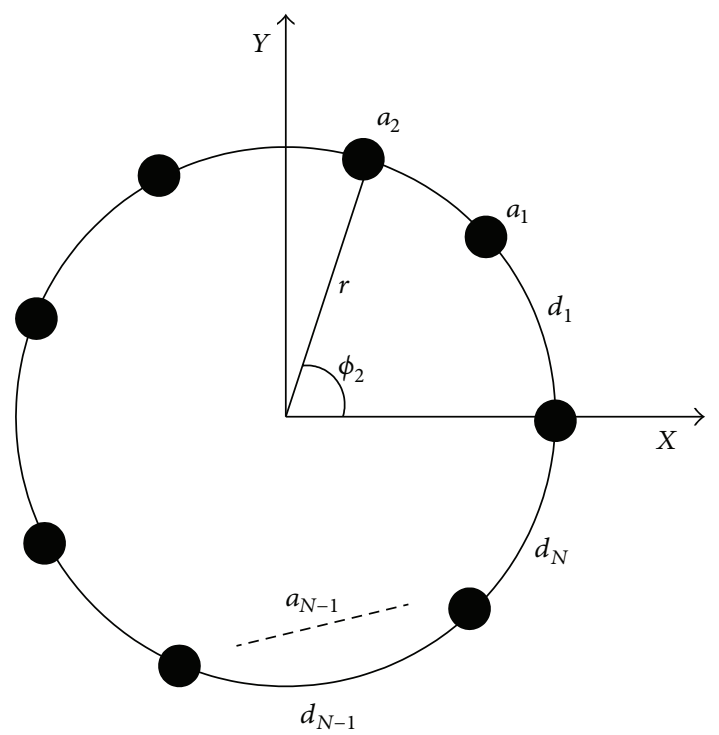

FIGURE 4: Geometry of a nonuniform circular antenna array with $N$ elements.

TABLE 4: Design variables obtained with CAIWO algorithm.

\begin{tabular}{lccc}
\hline $\begin{array}{l}\text { Number of } \\
\text { elements }\end{array}$ & FNBW & $d_{i}$ in terms of wavelength & Normalized $I_{n}$ \\
\hline \multirow{3}{*}{8} & $0.5600,1.0000$ & $0.4423,0.3059$ \\
& \multirow{2}{*}{70.27} & $1.0000,0.5734$ & $0.6405,0.7325$ \\
& & $0.9509,0.6363$ & $0.2203,0.1938$ \\
& $0.5503,0.5000$ & $0.3925,0.8590$ \\
\hline \multirow{4}{*}{55.85} & $0.5001,1.0000$ & $0.5823,0.3462$ \\
& & $0.9253,1.0000$ & $0.41608,0.7275$ \\
& & $0.5332,0.5313$ & $0.7032,0.0737$ \\
& & $0.9433,0.8756$ & $0.3758,0.3450$ \\
12 & $0.5567,0.5078$ & $0.4751,0.9033$ \\
\hline \multirow{3}{*}{46.26} & $0.6448,1.0000$ & $0.4958,0.4722$ \\
& & $1.0000,1.0000$ & $0.0010,0.4733$ \\
& & $0.6294,0.5000$ & $0.6587,0.8100$ \\
& & $0.5000,0.9564$ & $0.4700,0.4122$ \\
& & $0.5532,0.7578$ & $0.2060,0.4956$ \\
& & $1.0000,0.5967$ & $0.5455,0.9976$ \\
\hline
\end{tabular}

Then, the array factor can be simplifies as [13]

$$
A F(\phi)=\sum_{n=1}^{N} a_{n} \cdot e^{j k r\left(\cos \left(\phi-\phi_{n}\right)-\cos \left(\phi_{0}-\phi_{n}\right)\right)} .
$$

\section{Design Examples}

The first and most important parameter in antenna pattern synthesis is the normalized side-lobe level that is desired to be as low as possible. In this section, the CAIWO algorithm will be applied to determine the electrical and geometrical structure of circular antenna array for obtaining the radiation pattern with minimum SLL in two different cases. Three circular array antennas with $N=8,10$, and 12 elements are optimized in both cases.

4.1. Case One. The main goal of synthesis of antenna array in case one is to generate the radiation pattern with minimum side-lobe level for a specific first null beam width (FNBW). We have incorporated the maximum side-lobe level in addition to the average side lobe in the fitness function to ensure maximum directivity of the antenna arrays.

4.1.1. Fitness Function of Case One. The following objective functions represent these above requirements in a mathematical form [13]:

$$
\begin{aligned}
F_{\mathrm{NULL}}= & \left|A F\left(\phi_{\mathrm{NULL} 1}\right)\right|+\left|A F\left(\phi_{\mathrm{NULL} 2}\right)\right|, \\
F_{\mathrm{SLA}}= & \frac{1}{\pi+\phi_{\mathrm{NULL} 1}} \int_{-\pi}^{\phi_{\mathrm{NULL1}}}|A F(\phi)| d \phi \\
& +\frac{1}{\pi-\phi_{\mathrm{NULL} 2}} \int_{\phi_{\mathrm{NULL}}}^{\pi}|A F(\phi)| d \phi, \\
F_{\mathrm{MSL}}= & \left|A F\left(\phi_{\mathrm{MSLL} 1}\right)\right|+\left|A F\left(\phi_{\mathrm{MSL} 2}\right)\right|,
\end{aligned}
$$

where $\phi_{\text {NULL1 }}$ and $\phi_{\text {NULL2 }}$ are the two angle at the null and $\phi_{\text {MSLL1 }}$ and $\phi_{\text {MSLL2 }}$ are the angles where the maximum sidelobe level is obtained in the lower band $\left[-\pi, \phi_{\text {NULL1 }}\right]$ and higher band $\left[\phi_{\mathrm{NULL} 2}, \pi\right]$, respectively. Combining all of the above objectives, a final cost function can be formulated as follows [13]:

$$
F=\omega_{1} * F_{\mathrm{NULL}}+\omega_{2} * F_{\mathrm{SLA}}+\omega_{3} * F_{\mathrm{MSL}}
$$

where $\omega_{i}(i=1,2,3)$ represents the weights assigned to the functions. In order to emphasis the three objectives equally, we set $\omega_{i}$ that is equal to 1 . Since we are considering nonuniformly spaced circular array, the coupling will be different from one element to another [13]. This is especially critical as we are considering the radiation in the plane of antenna array. In order to get rid of the mutual coupling effect, we set the minimum value of arc separation between any two adjacent elements equal to $0.5 \lambda$. If $d_{i}$ in a solution $\left(d_{1}, d_{2}, \ldots, d_{n}, \ldots, d_{N}\right)$ is smaller than $0.5 \lambda$, this solution will be penalized by adding the following function to the cost function (9):

$$
F_{p}=\sum_{i}^{M}\left(0.5-d_{i}\right), \quad d_{i}<0.5 .
$$

$M$ is the total number of $d_{i}$ which is smaller than 0.5 in a solution.

4.1.2. Numerical Results. To illustrate the superiority of the CAIWO algorithm, three instantiations of the circular array antenna design problem are solved by using the CAIWO algorithm with five other state-of-the-art algorithms, namely MIWO, classical IWO, DE, PSO, and real coded GA. This DE variant is called $\mathrm{DE} / \mathrm{rand} / 1 / \mathrm{bin}$ and is the most widely used one in DE literature. 


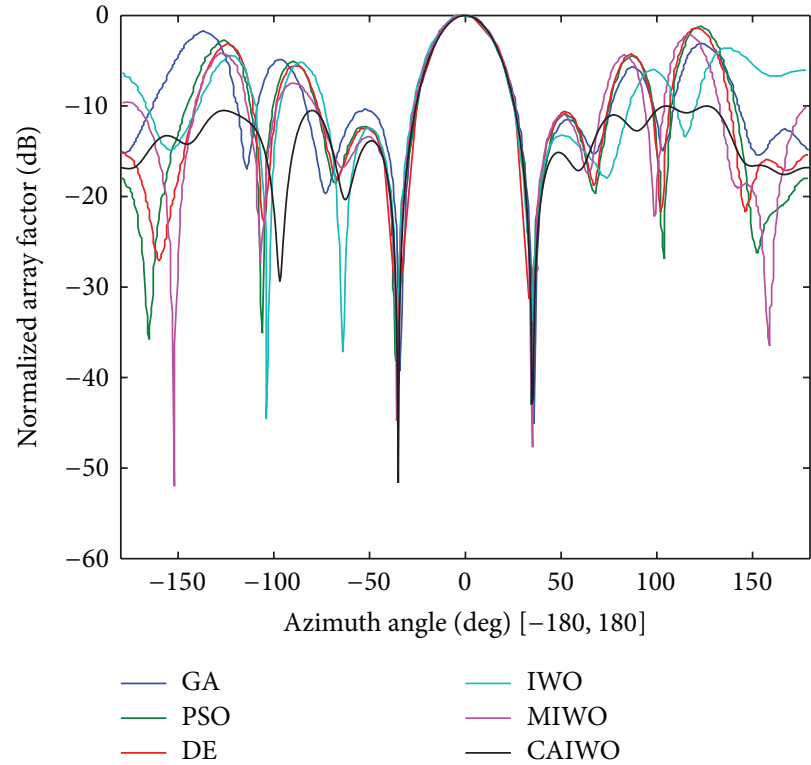

(a)

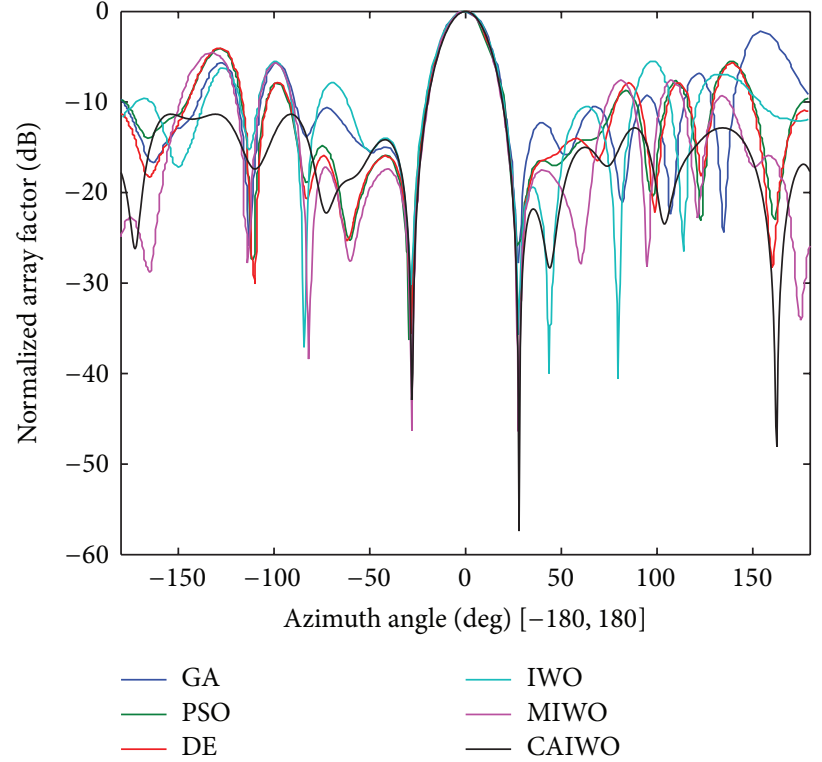

(b)

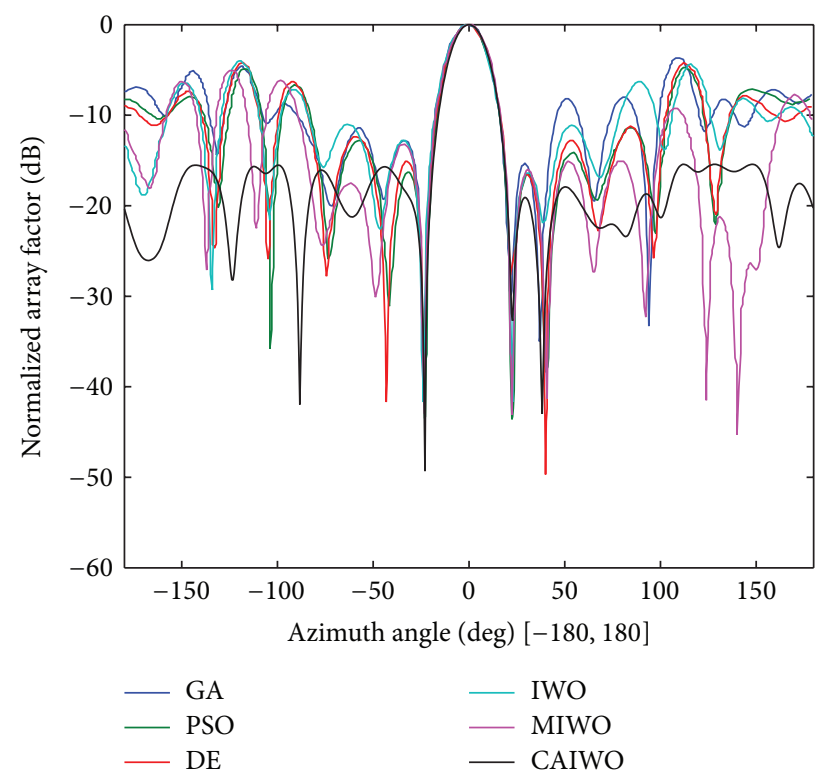

(c)

FIGURE 5: Normalized radiation patterns for circular arrays of different number of elements obtained using six different optimization algorithms. (a) For number of elements $N=8$. (b) For number of element $N=10$. (c) For number of elements $N=12$.

The three instantiations are arrays with 8,10 , and 12 elements. The FNBW is assumed to be a constant, corresponding to a uniform circular array with a uniform $0.5 \lambda$ spacing between the elements. The values of the element amplitudes are allowed to vary between $[0,1]$ (Table 3 ).

The control parameters for CAIWO were set through a series of parameter tuning experiments. The parameters for IWO, MIWO, PSO, DE, and GA were set following the guidelines provided in $[13,20]$. Once set, the values of parameters were used for each algorithm to solve all three simulation examples. The CAIWO, MIWO, and classical
IWO were let start from the same initial population over all runs on each problem.

Figure 5 presents the radiation patterns of the circular arrays optimized by CAWIO compared to other five algorithms in literature [13] (corresponding to the best of the 50 runs in each case) for 8,10 , and 12 element arrays. The normalized array geometry and current excitation weights are given in Table 4 . Figure 6 shows the convergence characteristic of CAIWO over three instances of the circular array design problem (corresponding to the best of the 50 runs in each case) for 8, 10, and 12 element arrays. In Table 5, the 
TABLE 5: Design figures of merit obtained in the best (out of 50) runs the six algorithms on three design instances.

\begin{tabular}{|c|c|c|c|c|c|}
\hline Number of elements & Algorithms & Mean SLL (dB) & $\operatorname{Max}$ SLL (dB) & Average null depth (dB) & Directivity $(\mathrm{dB})$ \\
\hline \multirow{6}{*}{8} & CAIWO & -13.158 & -10.01 & -46.88 & 9.67 \\
\hline & MIWO & -10.75 & -2.17 & -46.25 & 9.14 \\
\hline & IWO & -9.488 & -3.61 & -34.6 & 8.27 \\
\hline & $\mathrm{DE}$ & -10.34 & -1.35 & -32.95 & 8.87 \\
\hline & PSO & -10.23 & -1.17 & -40.6 & 8.78 \\
\hline & GA & -9.16 & -1.71 & -42.2 & 8.08 \\
\hline \multirow{6}{*}{10} & CAIWO & -16.95 & -11.42 & -50.16 & 12.48 \\
\hline & MIWO & -13.78 & -4.65 & -46.35 & 11.75 \\
\hline & IWO & -11.35 & -5.52 & -32.8 & 9.93 \\
\hline & $\mathrm{DE}$ & -12.46 & -4.07 & -34.4 & 10.79 \\
\hline & PSO & -12.15 & -4.07 & -31.1 & 10.55 \\
\hline & GA & -10.65 & -2.18 & -30 & 9.40 \\
\hline \multirow{6}{*}{12} & CAIWO & -18.76 & -15.43 & -41.015 & 12.85 \\
\hline & MIWO & -14.23 & -5.16 & -43.05 & 12.25 \\
\hline & IWO & -10.99 & -4.34 & -41.7 & 9.86 \\
\hline & $\mathrm{DE}$ & -11.66 & -4.17 & -31.4 & 10.39 \\
\hline & PSO & -11.61 & -4.75 & -43.55 & 10.37 \\
\hline & GA & -10.12 & -3.68 & -32.8 & 9.21 \\
\hline
\end{tabular}

TABLE 6: Comparisons of results obtained by CAIWO with other algorithms.

\begin{tabular}{|c|c|c|c|c|c|c|}
\hline Number of elements & Algorithms & Max SLL (dB) & HPBW $\left(^{\circ}\right)$ & ADR & Directivity $(\mathrm{dB})$ & Circumferences \\
\hline \multirow{6}{*}{8} & CAIWO & -13.0225 & 17.19 & 1.4974 & 9.6365 & 9.1210 \\
\hline & IWO & -8.1667 & 14.9 & 4.6579 & 8.0011 & 8.3999 \\
\hline & $\mathrm{BBO}$ & -12.24 & 18.6 & 5.9595 & 9.43 & 9.0710 \\
\hline & SA & -12.00 & 26.60 & 3.2383 & 10.7201 & 5.8750 \\
\hline & PSO & -10.7996 & 32 & 2.7910 & 7.8269 & 4.4931 \\
\hline & GA & -9.811 & 32 & 3.9417 & 7.6245 & 4.4054 \\
\hline \multirow{6}{*}{10} & CAIWO & -14.96 & 16.04 & 1.9514 & 10.2658 & 9.2333 \\
\hline & IWO & -10.8961 & 14.9 & 4.8786 & 9.5974 & 9.2637 \\
\hline & $\mathrm{BBO}$ & -13.95 & 16.60 & 2.6185 & 10.2032 & 9.2318 \\
\hline & SA & -13 & 18.4 & 3.2179 & 10.8722 & 8.0214 \\
\hline & PSO & -12.307 & 24.34 & 1.9755 & 8.6048 & 5.9029 \\
\hline & GA & -9.811 & 32 & 2.8140 & 9.8037 & 6.0886 \\
\hline \multirow{6}{*}{12} & CAIWO & -16.50 & 13.75 & 2.3018 & 10.7494 & 10.5351 \\
\hline & IWO & -11.0042 & 11.46 & 7.6718 & 9.9301 & 12.0781 \\
\hline & $\mathrm{BBO}$ & -14.372 & 14.8 & 2.2676 & 10.8448 & 10.6453 \\
\hline & SA & -13.91 & 19.60 & 3.2639 & 10.7069 & 7.9523 \\
\hline & PSO & -13.670 & 21.2 & 2.5265 & 9.3667 & 7.1419 \\
\hline & GA & -11.83 & 20.8 & 4.1439 & 10.2209 & 7.7724 \\
\hline
\end{tabular}

best results obtained (out of 50 independent runs) for the aforesaid three problem instances are judged in terms of the average SLL (in decibels), maximum SLL, the average null depth (in decibels), and the directivity (in decibels) for all the six algorithms based approaches.

From Figure 5, we can know that the radiation pattern synthesized by all these algorithms have same first null bandwidth (corresponding to the best of the 50 runs in each case) for 8, 10, and 12 element arrays, but the average SLLs in lower band and higher band obtained by CAIWO are better than those obtained by the other five algorithms [13].

From Tables 4 and 5, we can clearly state that CAIWO is much better in a statistically significant way than the other five metaheuristics algorithms, namely MIWO, IWO, PSO, $\mathrm{DE}$, and GA, on this specific case of circular array design. A scrutiny of Table 5 shows that the CAIWO obtains better 
TABLE 7: Comparison of results obtained by CAIWO with other algorithms for $N=8$ elements.

\begin{tabular}{llcccccccc}
\hline \multirow{2}{*}{ PSO [9] } & $I_{n}$ & 0.7765 & 0.3928 & 0.6069 & 0.8446 & 1 & 0.7015 & 0.9321 & 0.3583 \\
& $d_{n}$ & 0.359 & 0.5756 & 0.2494 & 0.7638 & 0.6025 & 0.8311 & 0.7809 & 0.3308 \\
\hline \multirow{2}{*}{ GA [9] } & $I_{n}$ & 0.3289 & 0.2537 & 0.7849 & 1 & 0.9171 & 0.5183 & 0.6176 & 0.4612 \\
& $d_{n}$ & 0.1739 & 0.3144 & 0.662 & 0.7425 & 0.6297 & 0.8929 & 0.4633 & 0.5267 \\
\hline \multirow{2}{*}{ SA [17] } & $I_{n}$ & 0.3047 & 0.484 & 0.7751 & 0.9867 & 0.3371 & 0.4422 & 0.4067 & 0.6807 \\
& $d_{n}$ & 0.9997 & 0.7743 & 0.9042 & 0.5652 & 0.8056 & 0.7818 & 0.5848 & 0.4594 \\
\hline \multirow{2}{*}{ BBO [9] } & $I_{n}$ & 1 & 0.6736 & 0.1678 & 1 & 0.9088 & 0.6553 & 0.7571 & 1 \\
& $d_{n}$ & 0.6341 & 1 & 1.8892 & 0.8456 & 0.5693 & 1.1639 & 1.3329 & 1.6367 \\
\hline \multirow{2}{*}{ IWO } & $I_{n}$ & 0.6188 & 0.3016 & 0.3394 & 0.337 & 0.2093 & 0.9749 & 0.3675 & 0.7029 \\
& $d_{n}$ & 1.6076 & 0.3132 & 1.1011 & 1.243 & 0.2924 & 1.9749 & 0.7915 & 1.0762 \\
\hline \multirow{2}{*}{ CAIWO } & $I_{n}$ & 0.9406 & 0.6999 & 0.6677 & 0.9998 & 0.9294 & 0.6882 & 0.8101 & 0.9710 \\
& $d_{n}$ & 0.5747 & 1.0263 & 1.7891 & 0.9507 & 0.5899 & 1.1968 & 1.3859 & 1.6077 \\
\hline
\end{tabular}

TABLE 8: Comparison of results obtained by CAIWO with other algorithms for $N=10$ elements.

\begin{tabular}{lcccccccccccc}
\hline \multirow{2}{*}{ PSO [9] } & $I_{n}$ & 1 & 0.7529 & 0.7519 & 1 & 0.5062 & 1 & 0.7501 & 0.7524 & 1 & 0.5067 \\
& $d_{n}$ & 0.317 & 0.9654 & 0.3859 & 0.9654 & 0.3185 & 0.3164 & 0.9657 & 0.3862 & 0.965 & 0.3174 \\
\hline \multirow{2}{*}{ GA [9] } & $I_{n}$ & 0.9545 & 0.4283 & 0.3392 & 0.9074 & 0.8086 & 0.4533 & 0.5634 & 0.6015 & 0.7045 & 0.5948 \\
& $d_{n}$ & 0.3641 & 0.4512 & 0.275 & 1.6373 & 0.6902 & 0.9415 & 0.4657 & 0.2898 & 0.6456 & 0.3282 \\
\hline \multirow{2}{*}{ SA [17] } & $I_{n}$ & 0.692 & 0.5679 & 0.5937 & 0.6703 & 0.9693 & 0.6014 & 0.3575 & 0.302 & 0.5908 & 0.9718 \\
& $d_{n}$ & 0.6221 & 0.988 & 0.7777 & 0.9934 & 0.6217 & 0.9514 & 0.7626 & 0.598 & 0.7655 & 0.941 \\
\hline \multirow{2}{*}{ BBO [9] } & $I_{n}$ & 1 & 1 & 1 & 0.3819 & 0.897 & 1 & 0.7679 & 0.8899 & 0.7246 & 1 \\
& $d_{n}$ & 0.5301 & 1.0603 & 1.3264 & 1 & 0.4307 & 0.4408 & 1.5276 & 1.3255 & 1 & 0.5904 \\
\hline \multirow{2}{*}{ IWO } & $I_{n}$ & 0.2851 & 0.5493 & 0.7386 & 0.1722 & 0.8401 & 0.2874 & 0.3323 & 0.7330 & 0.4616 & 0.4811 \\
& $d_{n}$ & 0.0100 & 0.5291 & 0.9957 & 1.2975 & 1.7320 & 0.5507 & 0.4642 & 0.7686 & 1.2974 & 1.6184 \\
\hline \multirow{2}{*}{ CAIWO } & $I_{n}$ & 0.9941 & 0.9842 & 0.9518 & 0.5123 & 0.9233 & 0.9858 & 0.6593 & 0.9196 & 0.7336 & 0.9997 \\
& $d_{n}$ & 0.5910 & 1.0471 & 1.2862 & 1.0304 & 0.4570 & 0.4422 & 1.4190 & 1.3552 & 1.0090 & 0.5962 \\
\hline
\end{tabular}

TABLE 9: Comparison of results obtained by CAIWO with other algorithms for $N=12$ elements.

\begin{tabular}{lcccccccccccccc}
\hline \multirow{2}{*}{ PSO [9] } & $I_{n}$ & 0.9554 & 0.6441 & 0.7109 & 0.7769 & 1 & 1 & 0.3958 & 0.7162 & 0.6746 & 0.7695 & 0.9398 & 0.6145 \\
& $d_{n}$ & 0.2569 & 0.8509 & 0.6607 & 0.7057 & 0.854 & 0.3734 & 0.1609 & 0.8321 & 0.6464 & 0.7079 & 0.833 & 0.26 \\
\hline \multirow{2}{*}{ GA [9] } & $I_{n}$ & 0.2064 & 0.5416 & 0.2246 & 0.6486 & 0.7212 & 0.7913 & 0.5277 & 0.3495 & 0.5125 & 0.4475 & 0.5233 & 0.8553 \\
& $d_{n}$ & 0.4936 & 0.4184 & 1.4474 & 0.7577 & 0.4204 & 0.5784 & 0.452 & 0.8872 & 0.7514 & 0.4202 & 0.4223 & 0.7234 \\
\hline \multirow{2}{*}{ SA [17] } & $I_{n}$ & 0.6231 & 0.399 & 0.3418 & 0.6054 & 0.9444 & 0.738 & 0.6741 & 0.3001 & 0.4311 & 0.5435 & 0.4195 & 0.9795 \\
& $d_{n}$ & 0.8315 & 0.791 & 0.6699 & 0.8087 & 0.7347 & 0.5331 & 0.4777 & 0.896 & 0.4874 & 0.8657 & 0.3461 & 0.5105 \\
\hline \multirow{2}{*}{ BBO [9] } & $I_{n}$ & 1 & 0.6501 & 0.6224 & 0.502 & 0.554 & 1 & 0.6683 & 0.7234 & 0.441 & 0.5123 & 0.4793 & 1 \\
& $d_{n}$ & 0.6704 & 1 & 1.3046 & 0.8081 & 1 & 0.431 & 0.6183 & 1.1574 & 1.3465 & 0.6551 & 1 & 0.6539 \\
\hline \multirow{2}{*}{ IWO } & $I_{n}$ & 0.8558 & 0.9912 & 0.4590 & 0.3337 & 0.7796 & 0.5693 & 0.1545 & 0.7573 & 0.3417 & 0.1292 & 0.7849 & 0.3379 \\
& $d_{n}$ & 0.5683 & 1.6654 & 0.2651 & 1.4680 & 1.1137 & 0.5021 & 0.3406 & 0.8223 & 0.7608 & 0.1292 & 1.6655 & 1.7793 \\
\hline \multirow{2}{*}{ CAIWO } & $I_{n}$ & 0.8996 & 0.7367 & 0.5923 & 0.4529 & 0.5664 & 0.9989 & 0.6111 & 0.7397 & 0.4340 & 0.5125 & 0.5364 & 0.9023 \\
& $d_{n}$ & 0.5700 & 1.0866 & 1.2745 & 0.7590 & 1.0124 & 0.4897 & 0.5611 & 1.1737 & 1.3395 & 0.6553 & 1.0571 & 0.5562 \\
\hline
\end{tabular}

result of four important merits: the average SLL, maximum SLL, null depth, and directivity in comparison to MIWO, IWO, PSO, DE, and GA for all instances.

4.2. Case Two. The goal of this case is to obtain the radiation pattern with minimum SLL and narrower beam width. This is done by manipulating the excitation currents and positions of elements. This case is considered similar to that reported in literatures $[7,9,10,17]$. The objective function to achieve the desired pattern using CAIWO is given as follows:

$$
\text { Fitness }=a_{1}\left|A F\left(\phi_{\mathrm{MSLL}}\right)\right|+a_{2} B W,
$$

where $a_{1}$ and $a_{2}$ are the weighting coefficients, $B W$ is the half power beam width of the array pattern measured in degrees, and $A F\left(\phi_{\mathrm{MSLL}}\right)$ is the maximum SLL. 


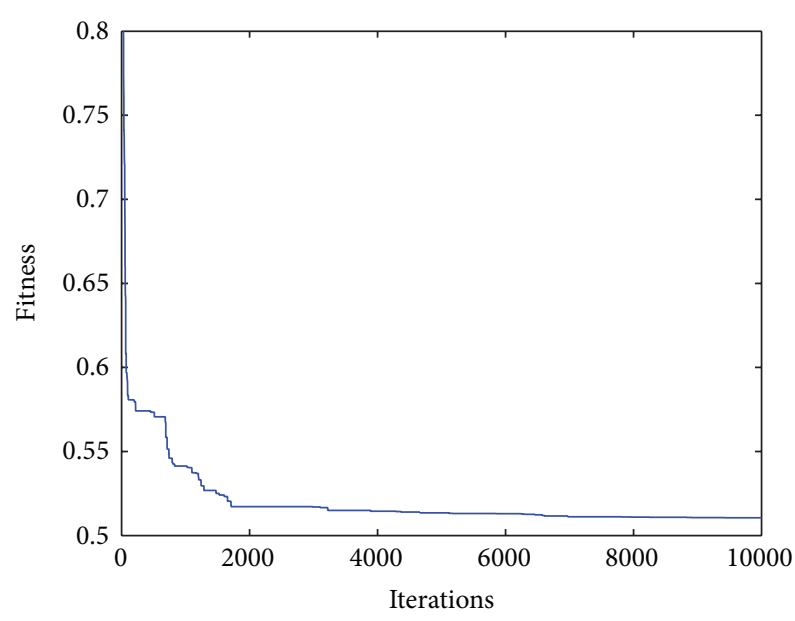

(a)

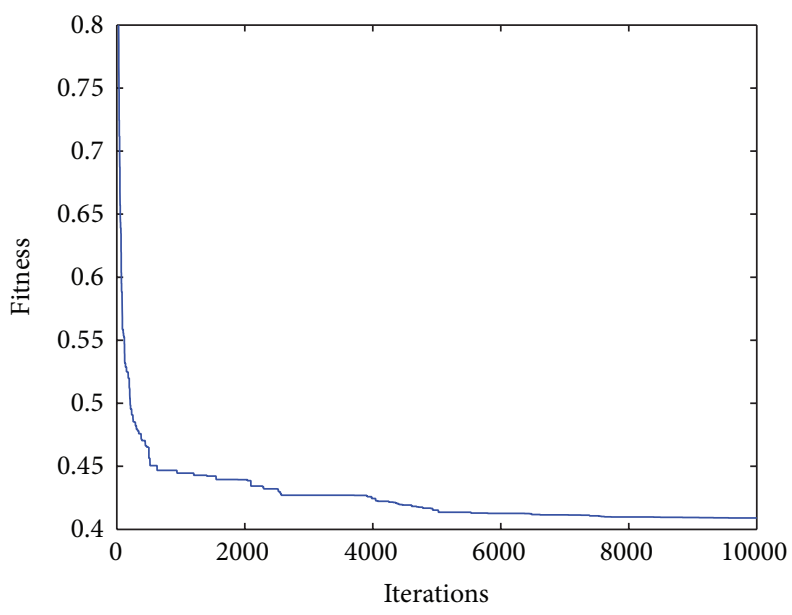

(b)

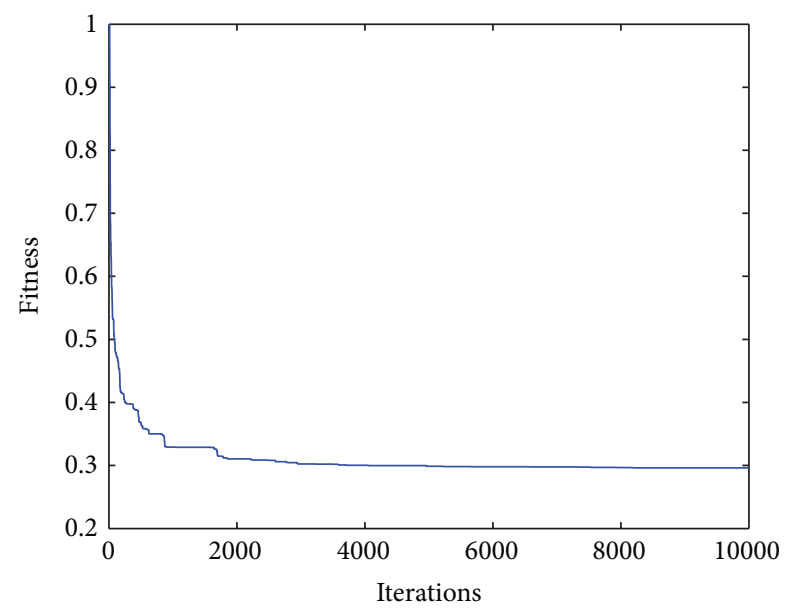

(c)

FIGURE 6: Convergence characteristic of six algorithms over three instances of the circular array design problem. (a) For number of elements $N=8$. (b) For number of element $N=10$. (c) For number of elements $N=12$.

In order to reduce the mutual coupling effects between elements, an additional term is added in the objective function (15). The ratio between the maximum and minimum excitation amplitudes is used to minimize the coupling effect. The minimization of the amplitude-excitation dynamic range (ADR) can reduce the mutual coupling problem [29]. The objective function can be expressed as follows:

$$
\text { Fitness }^{\prime}=a_{1}\left|A F\left(\phi_{\mathrm{MSLL}}\right)\right|+a_{2} B W+\mathrm{ADR},
$$

where $\mathrm{ADR}$ is the amplitude-dynamic ratio. The $\mathrm{ADR}$ is defined as the ratio between the maximum excitation amplitude and the minimum excitation amplitude. A small value of DRR not only can achieve a better control of the mutual coupling but also reduce the cost of the feeding network. The values of $a_{1}$ and $a_{2}$ are 70 and 1, respectively [7].

In this case, the values of the element amplitudes are allowed to vary between $[0,1]$ and the separation between $[0,2 \lambda]$, where $\lambda$ is the wavelength of the signal. The maximum number generation is 80 . The values of the rest parameters of CAIWO are same to those in Section 4.1. The stopping criterion for CAIWO is the maximum number of generations.

Along with the CAIWO results, the optimized array geometry and current excitation weights obtained using PSO [10], GA [9], SA [17], BBO [7], and IWO are also listed for comparisons in Tables 7, 8, and 9. In Table 6, the best results obtained for the aforesaid three problem instances are judged in terms of maximum SLL, HPBW, and ADR for all the six algorithms based approaches. Figure 7 presents the best radiation patterns of the circular arrays optimized by CAWIO compared to those of PSO, GA, SA, BBO, and IWO for 8, 10, and 12 element arrays.

In the 8 elements antenna array, the maximum SLL achieved by CAIWO is $-13.0225 \mathrm{~dB}$, the HPBW is $17.19^{\circ}$, and the ADR is 1.4974. Evidently, CAIWO provides better SLL, HPBW, and ADR than other techniques. The SLL obtained by CAIWO is lower by $0.7825,1.0225,3.2229,3.2115$ and $4.8558 \mathrm{~dB}$ than by the PSO, the GA, the SA, the BBO, and the IWO optimized arrays, respectively. The obtained HPBW is also narrower by $1.41,9.42,14.81$, and $14.81^{\circ}$ than the PSO, GA, 


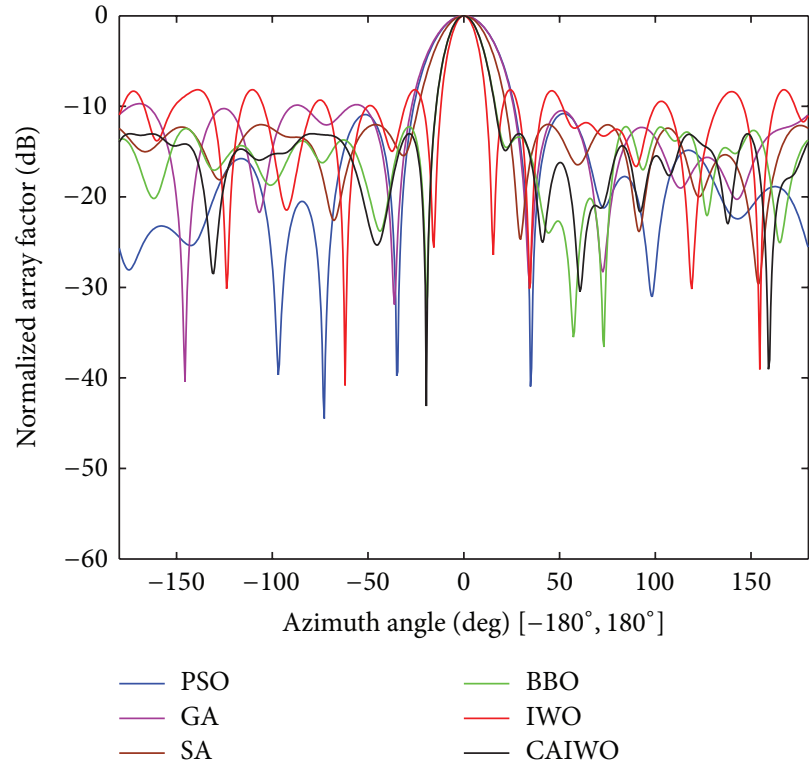

(a)

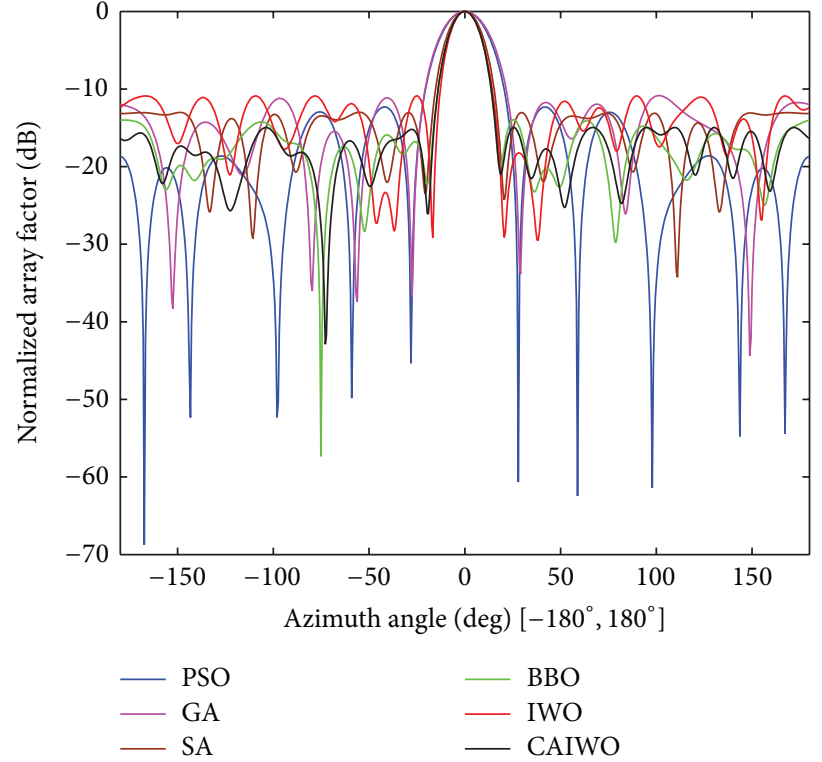

(b)

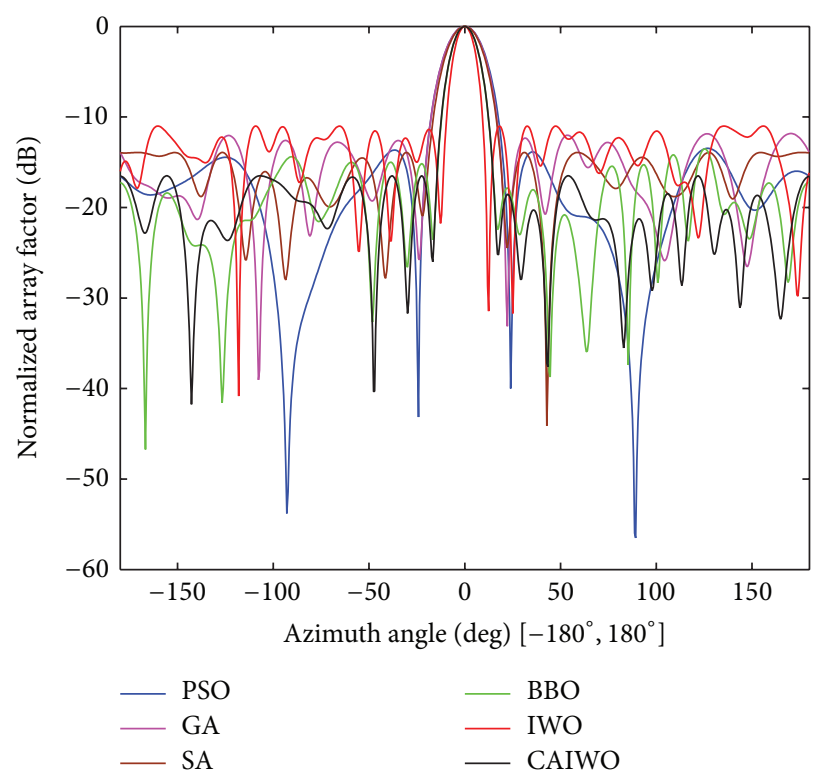

(c)

FIGURE 7: Normalized radiation patterns for circular arrays of different number of elements obtained using PSO GA SA BBO IWO and CAIWO. (a) For number of elements $N=8$. (b) For number of element $N=10$. (c) For number of elements $N=12$.

$\mathrm{SA}$, and $\mathrm{BBO}$ algorithms, respectively, except for the IWO algorithms. The ADR achieved by CAIWO is lower by 3.1605, $4.4621,1.7409,1.2936$, and 2.4443 than by the IWO, BBO, SA, PSO, and GA algorithms. The best radiation pattern of the 8 elements array generated by CAIWO is plotted in Figure 7(a) along with that of the PSO, GA, SA, BBO, and IWO algorithms.

The maximum SLL of 10 elements array achieved by CAIWO is $-14.96 \mathrm{~dB}$, the HPBW is $17.19^{\circ}$, and the ADR is 1.9514. Evidently, CAIWO outperforms the other techniques. The SLL obtained by CAIWO is lower by 1.01 1.96, 2.653, 5.149, and $4.0639 \mathrm{~dB}$ than by the PSO, the GA, the SA, the BBO, and the IWO optimized arrays, respectively. Moreover, the HPBW obtained by CAIWO is also narrower by $0.56,2.36,8.3$, and $15.96^{\circ}$ than the PSO, GA, SA, and BBO algorithms, respectively, except for the IWO algorithms. The ADR achieved by CAIWO is lower by $2.9272,0.6671,1.2665,0.0241$, and 0.8626 than by the IWO, BBO, SA, PSO, and GA algorithms. The best radiation pattern of the 10 elements array generated by CAIWO is plotted in Figure 7(b). For comparisons, the radiation patterns of the antennas generated by the PSO, GA, SA, BBO, and IWO algorithms are also drawn in Figure 7(b). The radiation pattern shows that CAIWO obtains excellent results. 
In the 12 elements antenna array, the maximum SLL achieved by CAIWO is $-16.5 \mathrm{~dB}$, the HPBW is $13.75^{\circ}$, and the ADR is 2.3018. Once again, the CAIWO algorithm yields results that are superior to the other algorithm. The maximum SLL is better than that achieved by the other algorithms. The reduction in SLL is significant and it is lower by $2.128,2.59,2.83,4.67$, and $5.4958 \mathrm{~dB}$ than by the $\mathrm{PSO}$, the GA, the SA, the BBO, and the IWO optimized arrays, respectively. The obtained HPBW is also better than that attained by the other algorithms. It is narrower by 1.05 , $5.85,7.45$, and $7.05^{\circ}$ than by the PSO, GA, SA, and BBO algorithms, respectively, except for the IWO algorithms. The ADR achieved by CAIWO is lower by 5.3700, 0.9621, 0.2247, and 1.8421 than by the IWO, SA, PSO, and GA algorithms, except for $\mathrm{BBO}$ algorithm. The best radiation pattern of the 12 elements array generated by CAIWO PSO, GA, SA, BBO, and IWO algorithms is plotted in Figure 7(c). The directivity and circumferences of optimized array are also listed in Table 6. The obtained circumferences of CAIWO are bigger than those of other algorithms, but it achieves higher directivity. Certainly, CAIWO again outperforms the other algorithms in obtaining the required antennas.

\section{Conclusions}

This paper proposed a variant invasive weed optimization called CAIWO algorithm. The proposed algorithm takes advantages of chaotic search and modified IWO with adaptive dispersion, where the seeds produced by a weed are dispersed in the search space with standard deviation specified by the fitness value of the weed. The statistical results obtained from the four benchmark functions demonstrate the superiority of the proposed CAIWO algorithm to MIWO and classical IWO. In addition, numerical examples of circular antenna array synthesis problems have been presented. We formulated two design problems with different purposes. The first case is an optimization task on the basis of a cost fitness that takes care of the average side-lobe levels and the null control. The second case takes care of the maximum SLL reduction with the constraint on the beam width. The fitness functions of both cases are minimized under a constraint in order to avoid the mutual coupling between the array elements. The simulation results over different element number show that the CAIWO algorithm could comfortably outperform the above mentioned algorithms in circular antenna array synthesis.

\section{Conflict of Interests}

The authors declare that there is no conflict of interests regarding the publication of this paper.

\section{References}

[1] M. M. Khodier and C. G. Christodoulou, "Linear array geometry synthesis with minimum sidelobe level and null control using particle swarm optimization," IEEE Transactions on Antennas and Propagation, vol. 53, no. 8, pp. 2674-2679, 2005.
[2] D. Marcano and F. Durán, "Synthesis of antenna arrays using genetic algorithms," IEEE Antennas and Propagation Magazine, vol. 42, no. 3, pp. 12-20, 2000.

[3] E. Rajo-Iglesias and O. Quevedo-Teruel, "Linear array synthesis using an ant-colony-optimization-based algorithm," IEEE Antennas and Propagation Magazine, vol. 49, no. 2, pp. 70-79, 2007.

[4] K.-K. Yan and Y. Lu, "Sidelobe reduction in array-pattern synthesis using genetic algorithm," IEEE Transactions on Antennas and Propagation, vol. 45, no. 7, pp. 1117-1122, 1997.

[5] A. Tennant, M. M. Dawoud, and A. P. Anderson, "Array pattern nulling by element position perturbations using a genetic algorithm," Electronics Letters, vol. 30, no. 3, pp. 174-176, 1994.

[6] F. J. Ares-Pena, J. A. Rodriguez-Gonzalez, E. Villanueva-Lopez, and S. R. Rengarajan, "Genetic algorithms in the design and optimization of antenna array patterns," IEEE Transactions on Antennas and Propagation, vol. 47, no. 3, pp. 506-510, 1999.

[7] U. Singh and T. S. Kamal, "Design of non-uniform circular antenna arrays using biogeography-based optimisation," IET Microwaves, Antennas and Propagation, vol. 5, no. 11, pp. 13651370, 2011.

[8] C. A. Balanis, Antenna Theory: Analysis and Design, John Wiley \& Sons, New York, NY, USA, 2012.

[9] M. A. Panduro, A. L. Mendez, R. Dominguez, and G. Romero, "Design of non-uniform circular antenna arrays for side lobe reduction using the method of genetic algorithms," $A E U$ International Journal of Electronics and Communications, vol. 60, no. 10, pp. 713-717, 2006.

[10] M. Shihab, Y. Najjar, N. Dib, and M. Khodier, "Design of non-uniform circular antenna arrays using particle swarm optimization," Journal of Electrical Engineering, vol. 59, no. 4, pp. 216-220, 2008.

[11] M. A. Panduro, C. A. Brizuela, L. I. Balderas, and D. A. Acosta, "A comparison of genetic algorithms, particle swarm optimization and the differential evolution method for the design of scannable circular antenna arrays," Progress in Electromagnetics Research B, no. 13, pp. 171-186, 2009.

[12] L. Gürel and Ö. Ergül, "Design and simulation of circular arrays of trapezoidal-tooth log-periodic antennas via genetic optimization," Progress in Electromagnetics Research, vol. 85, pp. 243-260, 2008.

[13] G. G. Roy, S. Das, P. Chakraborty, and P. N. Suganthan, "Design of non-uniform circular antenna arrays using a modified invasive weed optimization algorithm," IEEE Transactions on Antennas and Propagation, vol. 59, no. 1, pp. 110-118, 2011.

[14] A. Mandal, H. Zafar, S. Das, and A. V. Vasilakos, "Efficient circular array synthesis with a memetic differential evolution algorithm," Progress in Electromagnetics Research B, no. 38, pp. 367-385, 2012.

[15] P. Ghosh, J. Banerjee, S. Das, and S. S. Chaudhury, "Design of non-uniform circular antenna arrays-an evolutionary algorithm based approach," Progress in Electromagnetics Research B, no. 43, pp. 333-354, 2012.

[16] P. Civicioglu, "Circular antenna array design by using evolutionary search algorithms," Progress in Electromagnetics Research B, vol. 54, pp. 265-284, 2013.

[17] M. Rattan, M. S. Patterh, and B. S. Sohi, "Optimization of circular antenna arrays of isotropic radiators using simulated annealing," International Journal of Microwave and Wireless Technologies, vol. 1, no. 5, pp. 441-446, 2009. 
[18] A. R. Mehrabian and C. Lucas, "A novel numerical optimization algorithm inspired from weed colonization," Ecological Informatics, vol. 1, no. 4, pp. 355-366, 2006.

[19] A. Foudazi and A. R. Mallahzadeh, "Pattern synthesis for multifeed reflector antennas using invasive weed optimisation," IET Microwaves, Antennas and Propagation, vol. 6, no. 14, pp. 15831589, 2012.

[20] S. Karimkashi and A. A. Kishk, "Invasive weed optimization and its features in electromagnetics," IEEE Transactions on Antennas and Propagation, vol. 58, no. 4, pp. 1269-1278, 2010.

[21] S. H. Sedighy, A. R. Mallahzadeh, M. Soleimani, and J. RashedMohassel, "Optimization of printed yagi antenna using invasive weed optimization (IWO)," IEEE Antennas and Wireless Propagation Letters, vol. 9, pp. 1275-1278, 2010.

[22] Y.-Y. Bai, S. Xiao, C. Liu, and B.-Z. Wang, "A hybrid IWO/PSO algorithm for pattern synthesis of conformal phased arrays," IEEE Transactions on Antennas and Propagation, vol. 61, no. 4, pp. 2328-2332, 2013.

[23] S. Karimkashi, A. A. Kishk, and D. Kajfez, "Antenna array optimization using dipole models for MIMO applications," IEEE Transactions on Antennas and Propagation, vol. 59, no. 8, pp. 3112-3116, 2011.

[24] A. R. Mallahzadeh, H. Oraizi, and Z. Davoodi-Rad, "Application of the invasive weed optimization technique for antenna configurations," Progress in Electromagnetics Research, vol. 79, pp. 137-150, 2008.

[25] F. M. Monavar, N. Komjani, and P. Mousavi, "Application of invasive weed optimization to design a broadband patch antenna with symmetric radiation pattern," IEEE Antennas and Wireless Propagation Letters, vol. 10, pp. 1369-1372, 2011.

[26] H. Wu and C. Li, "Planar array synthesis with sidelobe reduction and null control using invasive weed optimization," Progress in Electromagnetics Research M, vol. 33, pp. 83-94, 2013.

[27] M. Ahmadi and H. Mojallali, "Chaotic invasive weed optimization algorithm with application to parameter estimation of chaotic systems," Chaos, Solitons \& Fractals, vol. 45, no. 9-10, pp. 1108-1120, 2012.

[28] P. N. Suganthan, N. Hansen, J. J. Liang et al., "Problem definitions and evaluation criteria for the CEC 2005 special session on real-parameter optimization," KanGAL Report 2005005, 2005.

[29] J. A. Rodrfguez, F. Ares, and E. Moreno, "Linear array pattern synthesis optimizing array element excitations using the simulated annealing technique," Microwave and Optical Technology Letters, vol. 23, no. 4, pp. 224-226, 1999. 


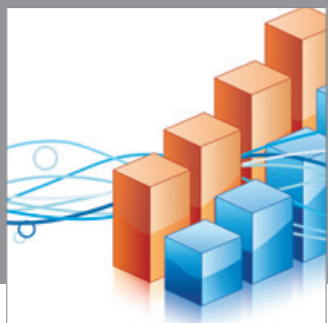

Advances in

Operations Research

mansans

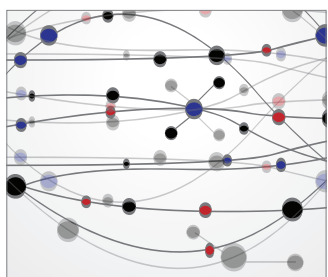

The Scientific World Journal
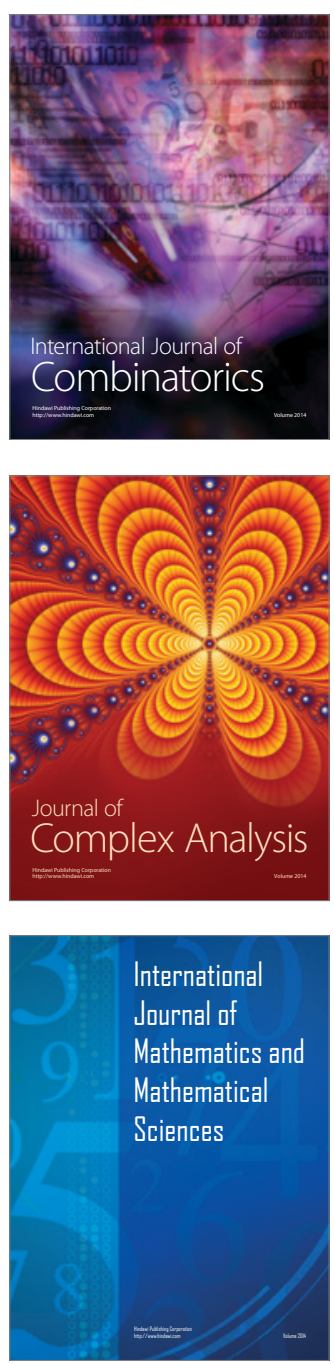
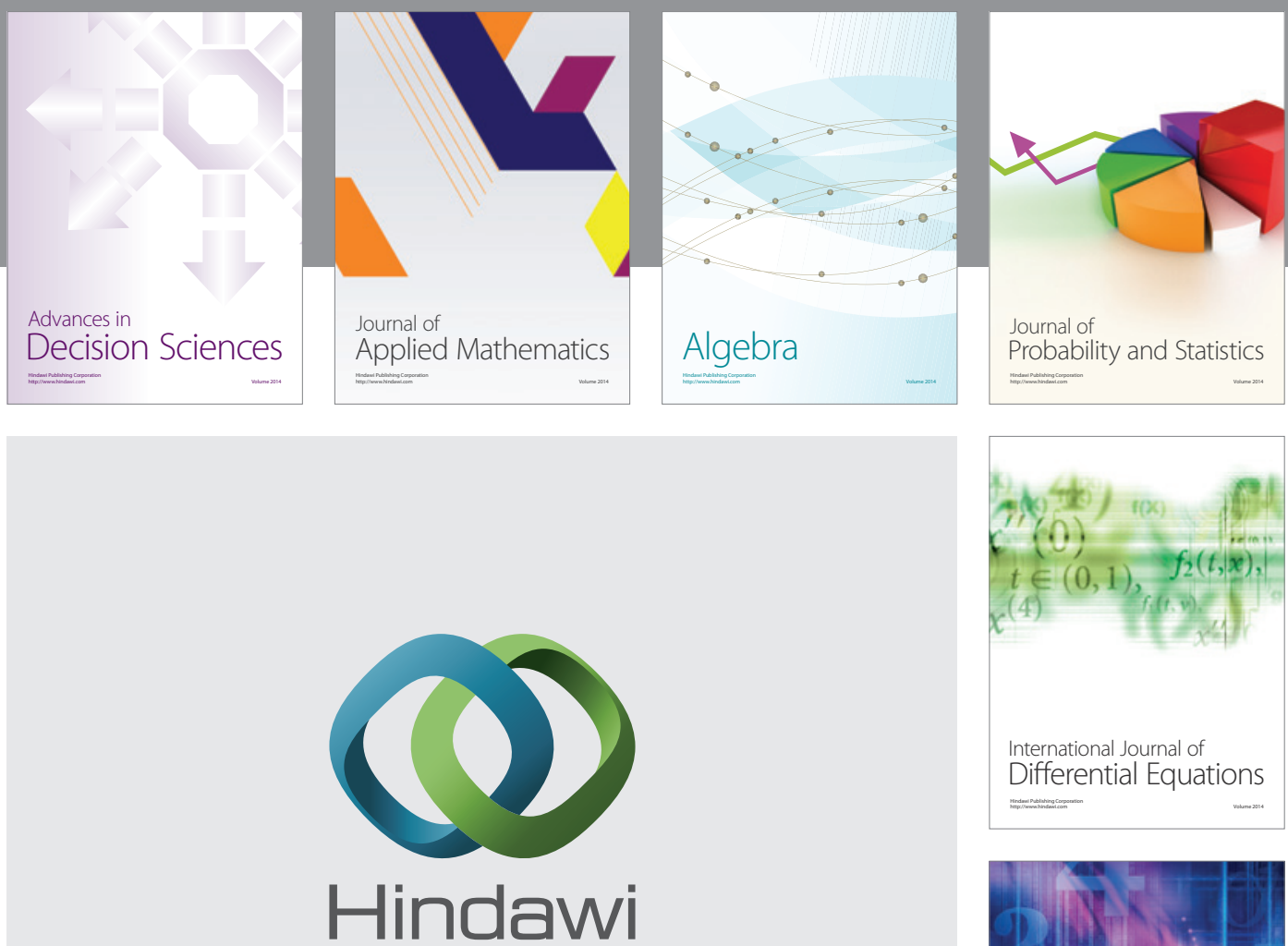

Submit your manuscripts at http://www.hindawi.com
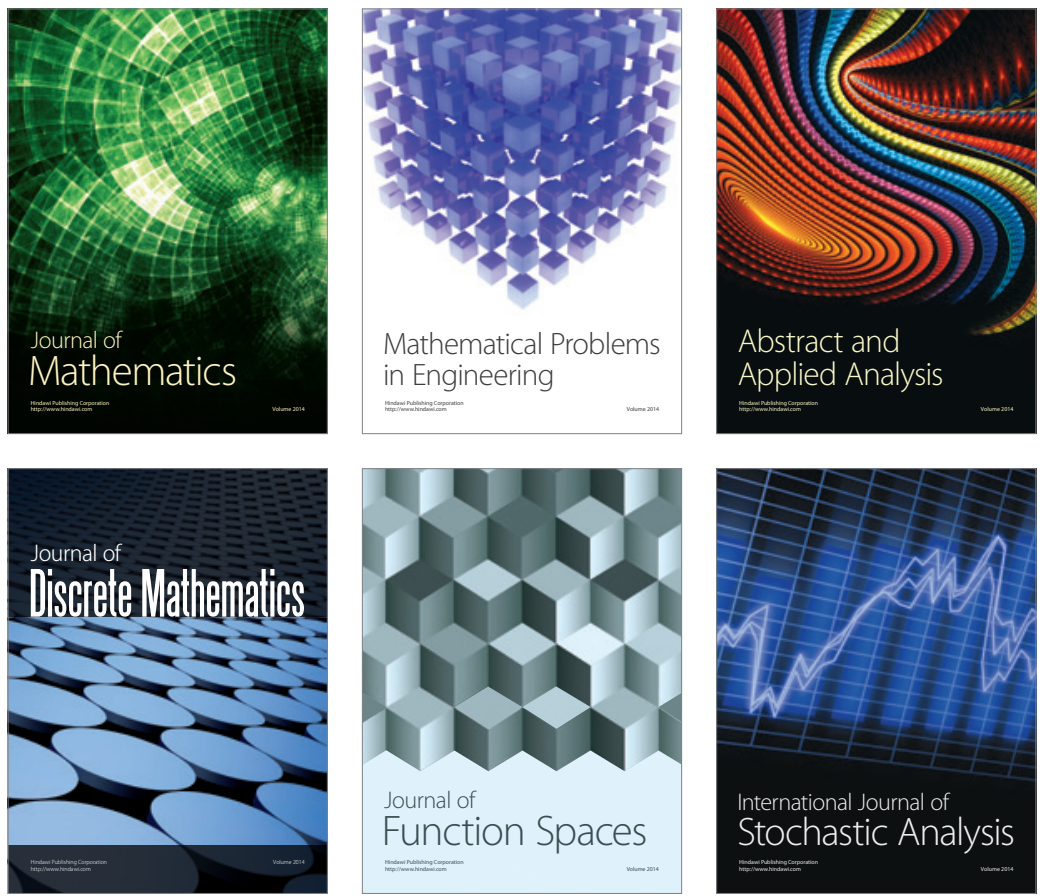

Journal of

Function Spaces

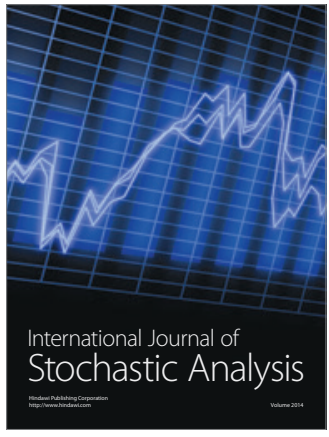

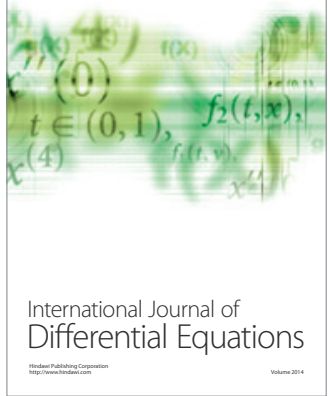
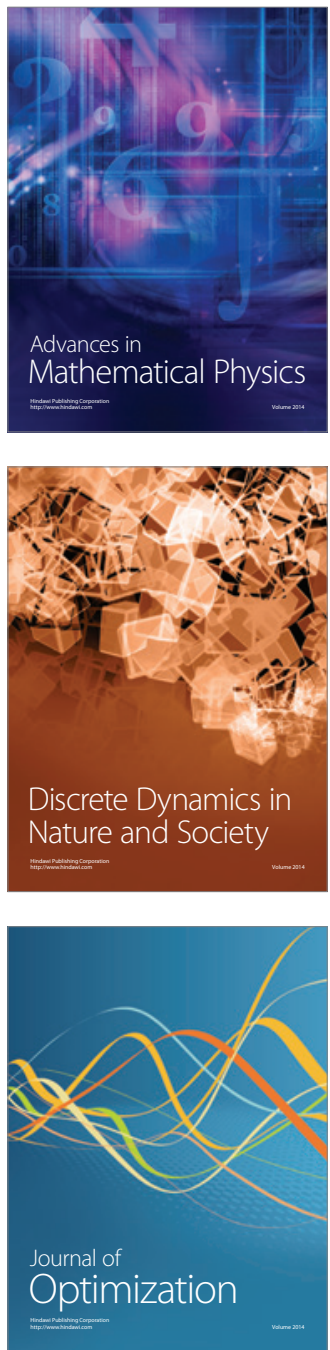